\title{
The Lady Windermere Syndrome
}

\author{
Jeffrey Forris Beecham Chick • Nikunj Rashmikant Chauhan • \\ Ryan James Bair · Vishal Rashmikant Chauhan
}

Received: 13 September 2012/ Accepted: 10 October 2012/Published online: 25 October 2012

(C) SIMI 2012

\section{Case}

A 78-year-old woman with bronchiectasis, numerous prior admissions for bacterial pneumonia, and mitral valve prolapse presented to this hospital with fever and cough for 6 days. The patient reported developing a nagging, persistent cough described as "inhaling cake crumbs," without sputum production 6 days prior. Concomitantly, the patient experienced low-grade temperatures ranging from $37.8-38.6^{\circ} \mathrm{C}$. On presentation to this hospital, the patient reported worsening "coughing attacks," complicated by diffuse myalgias and generalized weakness. The patient, however, denied recent illnesses or travel. Physical examination was notable for a temperature of $38.9^{\circ} \mathrm{C}$, respirations of 29 breaths per minute, an oxygen saturation of $90 \%$ while breathing ambient air, and diffuse wheezing and rhonchi throughout all the lung fields. A chest radiograph showed widespread bronchiectasis bilaterally, but no consolidative opacities, pleural effusions, pulmonary edema, or pneumothoraces (Fig. 1). Computed tomography of the chest without intravenous contrast material demonstrated moderate bilateral bronchiectasis as well as tree-in-bud nodularity throughout the right, middle and lingular segments, suggestive of

J. F. B. Chick $(\bowtie)$. N. R. Chauhan

Department of Radiology, Brigham and Women's Hospital, Harvard Medical School, Boston, MA, USA

e-mail: jchick@partners.org

R. J. Bair

Department of Radiation Oncology, University of Chicago Medical Center, Pritzker School of Medicine, Chicago, IL, USA

V. R. Chauhan

Department of Medicine, University Hospitals, Case Western Reserve University School of Medicine, Cleveland, OH, USA mycobacterium avium-intracellulare infection (Fig. 2). Two separate sputum samples were obtained, and were subsequently positive for mycobacterium avium-intracellulare. Human immunodeficiency virus testing was negative. The patient was initially prescribed ceftazidime and levofloxacin with transition to clarithromycin, rifampin, and ethambutol for 1 year, with complete resolution of her symptoms. Repeated computed tomography of the chest without intravenous contrast material completed 6 months later demonstrated persistent, moderate bilateral bronchiectasis with near complete resolution of the previously seen tree-in-bud nodularity, suggestive of resolving mycobacterium avium-intracellulare infection (Fig. 3).

\section{Discussion}

The Lady Windermere Syndrome, which was first described in 1992, comprises a spectrum of reticulonodular opacities, tree-in-bud nodularity, and cavitary lesions superimposed on cylindrical bronchiectasis of the right, middle lobe and lingular segments due to pulmonary mycobacterium avium-intracellulare infection $[1,2]$. The syndrome, which was named after a fussy character in Oscar Wild's Lady Windermere's Fan, is frequently found in elderly women with scoliosis, pectus excavatum, and mitral valve prolapse, without severe cardiopulmonary disease or significant smoking history [1]. In individuals with such attributes or characteristics, it is theorized that voluntary cough suppression leads to decreased clearance from the angulated right middle lobe and lingular bronchi, predisposing individuals to mycobacterium avium-intracellulare infection [1]. Although controversy exists regarding the treatment of pulmonary mycobacterium avium-intracellulare infection, the typical treatment regimen consists of clarithromycin, 


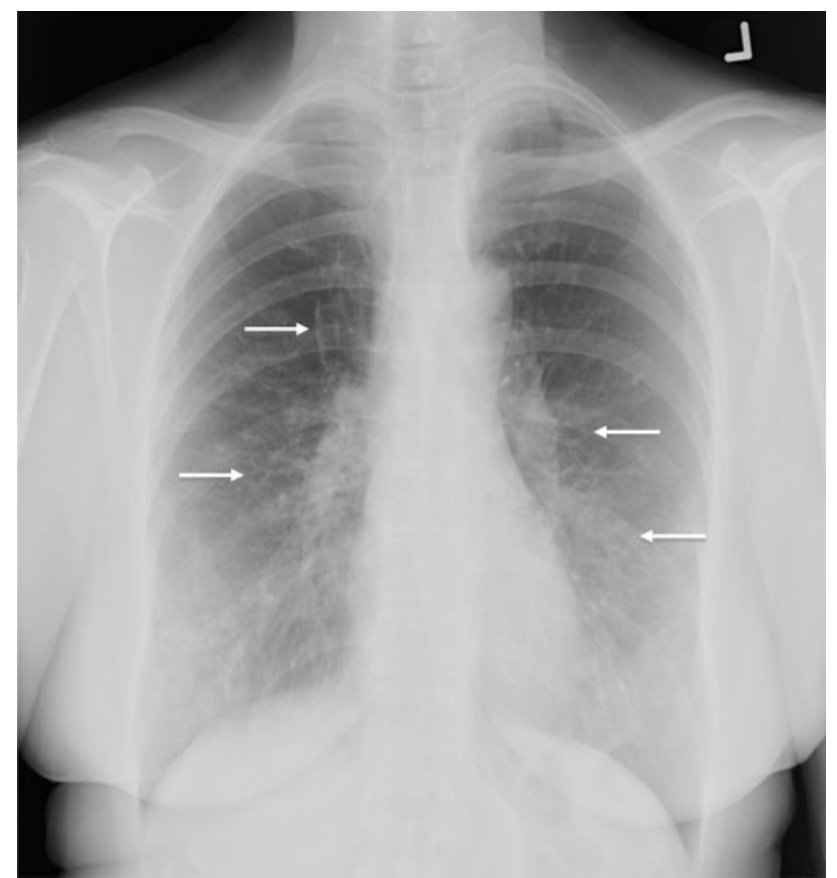

Fig. 1 Single posteroanterior chest radiograph demonstrating widespread bronchiectasis (arrows), without consolidative opacities, pleural effusions, pulmonary edema, or pneumothoraces

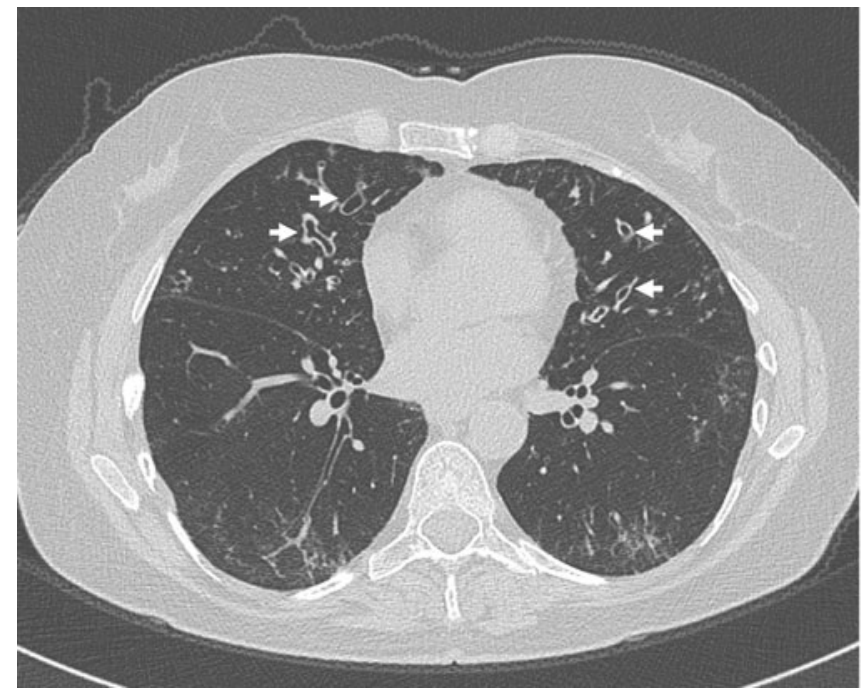

Fig. 2 Two axial computed tomographic images without intravenous contrast material in lung windows demonstrating extensive bilateral bronchiectasis (arrows) with superimposed tree-in-bud nodularity of

rifampin, and ethambutol for 1-2 years [3]. This case illustrates the classic chest radiograph and computed tomography findings of The Lady Windermere Syndrome.

\section{Conflict of interest None.}

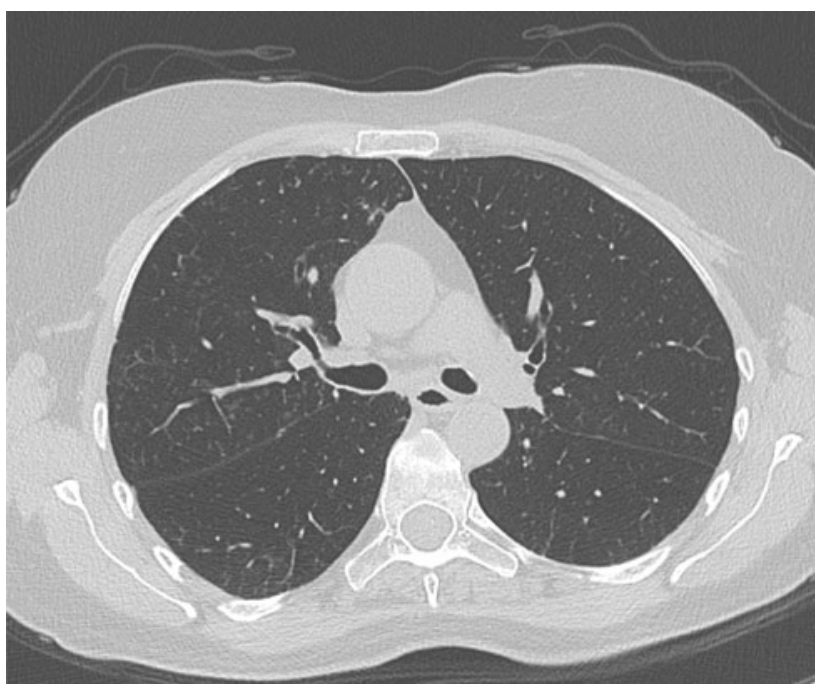

Fig. 3 Single axial computed tomographic image without intravenous contrast material in lung windows demonstrating near complete resolution of the previously seen tree-in-bud nodularity in the right middle and lingular segments, suggestive of resolving mycobacterium avium-intracellulare infection

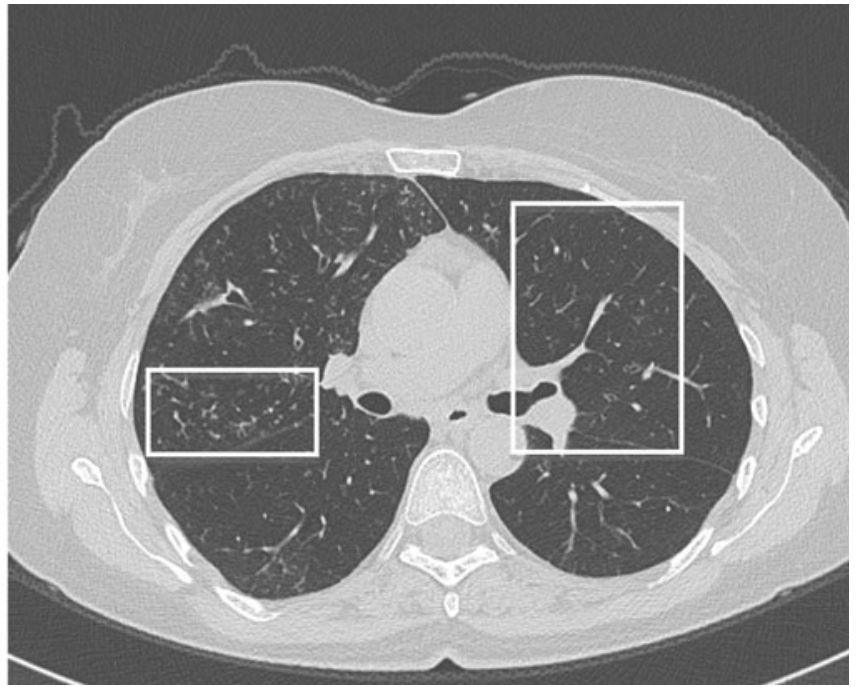

the right, middle and lingular segments (squares), suggestive of mycobacterium avium-intracellulare infection and the Lady Windermere Syndrome

\section{References}

1. Reich JM, Johnson RE (1992) Mycobacterium avium complex pulmonary disease presenting as an isolated lingular or middle lobe pattern. The Lady Windermere syndrome. Chest 101(6):1605-1609 
2. Prince DS, Peterson DD, Steiner RM et al (1989) Infection with mycobacterium avium complex in patients without predisposing conditions. NEJM 321(13):863-868
3. Griffith DE, Aksamit T, Brown-Elliot BA et al (2007) An official ATS/ IDSA statement diagnosis, treatment, and prevention of nontuberculous mycobacterial diseases. Am J Respir Crit Care Med 175:367-416 